\title{
INVESTIGATION INTO THE BEHAVIOUR AND MODELLING OF CHROMATIC ABERRATIONS IN NON-METRIC DIGITAL CAMERAS
}

\author{
Derek D Lichti ${ }^{1}$, David Jarron ${ }^{1}$, Mohzdeh Shahbazi ${ }^{1}$, Petra Helmholz ${ }^{2}$ and Robert Radovanovic ${ }^{3}$ \\ ${ }^{1}$ Department of Geomatics Engineering, The University of Calgary, Calgary, Canada - ddlichti@ucalgary.ca, \\ dmjarron@ucalgary.ca, mozhdeh.shahbazi@ucalgary.ca \\ ${ }^{2}$ Spatial Sciences, School for Earth and Planetary Sciences, Curtin University, Perth, Australia - petra.helmholz@curtin.edu.au \\ ${ }^{3}$ McElhanney Ltd, Calgary, Canada - rradovanovic@mcelhanney.com
}

Technical Commission II

KEY WORDS: camera self-calibration, chromatic aberration, parameter constraints

\begin{abstract}
:
Chromatic aberration in colour digital camera imagery can affect the accuracy of photogrammetric reconstruction. Both longitudinal and transverse chromatic aberrations can be effectively modelled by making separate measurements in each of the blue, green and red colour bands and performing a specialized self-calibrating bundle adjustment. This paper presents the results of an investigation with two aims. The first aim is to quantify the presence of chromatic aberration in two sets of cameras: the six individual cameras comprising a Ladybug5 system, calibrated simultaneously in air; and four GoPro Hero 5 cameras calibrated independently under water. The second aim is to investigate the impacts of imposing different constraints in the self-calibration adjustment. To this end, four different adjustment cases were performed for all ten cameras: independent adjustment of the observations from each colour band; combined adjustment of all colour bands' observations with common object points; combined adjustment of all colour bands with common object points and common exterior orientation parameters for each colour band triplet; and combined adjustment with common object points and certain common interior orientation parameters. The results show that the Ladybug5 cameras exhibit a small (1-2 pixel) amount of transverse chromatic aberration but no longitudinal chromatic aberration. The GoPro Hero 5 cameras exhibit significant (25 pixel) transverse chromatic aberration as well as longitudinal chromatic aberration. The principal distance was essentially independent of the adjustment case for the Ladybug5, but it was not for the GoPro Hero 5. The principal point position and precision were both affected considerably by adjustment case. Radial lens distortion was invariant to the adjustment case. The impact of adjustment case on decentring distortion was minimal in both cases.
\end{abstract}

\section{INTRODUCTION}

\subsection{Background}

Colour digital cameras are routinely used to make threedimensional measurements from imagery using photogrammetric methods for a vast range of applications. As reviewed by Luhmann et al (2006), several means exist to produce colour imagery with solid-state imaging sensors. Colour filters arrays such as the Bayer filter are the most common.

It is well known that the imagery captured with colour digital cameras can be affected by imaging distortions, including chromatic aberration. Chromatic aberration is caused by the dispersion of light by the lens assembly. Two types of chromatic aberration exist. Longitudinal chromatic aberration results in a wavelength-dependent focal length where the focal length increases with wavelength (Ray, 1994). Transverse chromatic aberration causes wavelength dependence of radial lens distortion (Luhmann et al, 2006).

Several examples of chromatic aberration exist in the photogrammetric literature. Both Van den Heuvel et al (2006) and Pöntinen (2008) report chromatic aberration in fisheye lens systems. In addition, Menna et al (2017) and Helmholz and Lichti (2019) both report the presence of chromatic aberration effects in underwater imagery captured with a camera system coupled with a planar housing port. The common thread among these examples is the presence of chromatic aberration effects in imagery produced by a colour filter array sensor and broadband illumination. Robson et al (2014) describe an experiment in which the illumination reaching the sensor is controlled by fitting the camera system with different narrow band interference filters. They report wavelength dependencies in the interior orientation parameters determined by self-calibrating bundle adjustment.

Depending on the accuracy required for a particular photogrammetric measurement application, the effects of chromatic aberration must be mitigated. At least two options exist for mitigating errors due to chromatic aberrations (Cronk et al, 2006): modelling the effect with colour-dependent calibration parameters; and correction by image pre-processing. One approach for modelling chromatic aberration is based on the self-calibrating bundle adjustment (Luhmann et al., 2006). Colour composite images of a calibration target field are captured in a strong network configuration. Each colour image is separated into red (R), green $(\mathrm{G})$ and blue (B) channel images. The image coordinates of the target centres are observed in each set of separated colour-band images. Each colour image triplet is modelled with common exterior orientation parameters, but separate sets of interior orientation parameters (a virtual camera; Luhmann et al., 2006) that include the principal distance, the principal point and lens distortion parameters. The effectiveness of this approach in terms of improving both precision and accuracy is demonstrated on SLR cameras by Luhmann et al (2006). 


\subsection{Objectives}

The purpose of this paper is two-fold. First, it reports on the chromatic aberration that can be observed in two types of camera systems. The first is the Ladybug5 camera system, which comprises six integrated cameras in a rigid housing. It finds application in mobile mapping and robotics. The Ladybug5 image in Figure 1a shows transverse chromatic aberration at the target edges at the level of 1-2 pixels. As will be described, this system has been calibrated over a test field of targets in a controlled environment. A preliminary report of chromatic aberration effects in this system is given by Jarron et al (2019a). Extensively-detailed analyses are provided herein.

The second camera system is a set of four GoPro Hero 5 Black cameras. These cameras are used for a wide range of applications ranging from archaeology to UAV mapping to underwater photogrammetry. Each camera was sealed in an acrylic housing and independently calibrated underwater in a fresh water tank. Underwater imagery captured with these cameras exhibits a much higher degree (up to 25 pixels) of transverse chromatic aberration (Figure 1b).

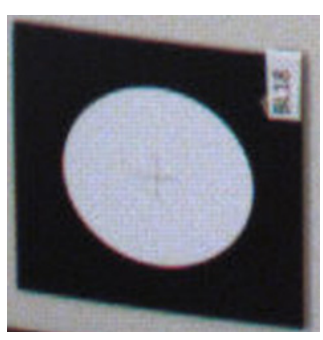

a)

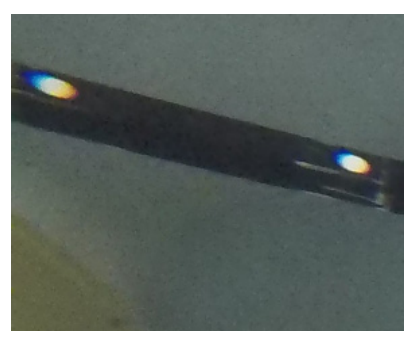

b)
Figure 1. Chromatic aberration in a) a Ladybug5 image and b) an underwater GoPro Hero 5 image.

The second objective of this study is to quantify the effectiveness of several approaches to model the chromatic aberrations. All are based on the acquisition of images of a target field in a well-designed network configuration, separation of the imagery into R, G and B images, and free-network, selfcalibrating bundle adjustment. Each case incorporates different types of geometric constraints within the self-calibrating bundle adjustment. The cases investigated are:

Case 1. Independent adjustment of the observations of each colour band. That is, separate adjustments of the red, green and blue observations.

Case 2. Adjustment with common object points. In other words, a combined adjustment of observations from all three bands. Three sets of interior orientation parameters (one per colour band) are estimated for each camera. Moreover, separate exterior orientation parameters are estimated for each colour band image.

Case 3. Adjustment with common exterior orientation parameters. That is, the combined adjustment with added constraints to force the red, green and blue exterior orientation parameters to be equal.

Case 4. Adjustment with selected common interior orientation parameters. This is the Case 2 adjustment with constraints to enforce the equivalence of the principal point coordinates of the cameras corresponding to the three spectral bands. In principle, these parameters should be unaffected by chromatic aberration.
Whereas Case 3 is akin to the approach of Luhmann et al. (2006), the other three represent relaxed versions. It should be noted that the modelling approaches listed above are similar to those reported by Matsuoka et al (2012). However, their paper focuses more on other image-space transformation methods for correcting chromatic aberration. For the bundle adjustment methods, they report only image point displacements between red and green bands and the resulting interior orientation or exterior orientation parameters are not presented.

This paper is organized as follows. Section 2 presents the functional models for each adjustment case investigated. Details of the experiments performed for both camera systems are given in Section 3. Results are presented and analysed in detail in Section 4 and Section 5 provides conclusions and recommendations for future work.

\section{METHODOLOGY}

\subsection{Image Geometry Models}

Both camera systems studied herein are fitted with wide-angle lenses. Imagery captured with these cameras in air exhibits significant barrel distortion. In a recent investigation, the application of fisheye lens models to Ladybug5 and GoPro Hero 5 imagery is reported (Jarron et al, 2019c). For this investigation, however, the modelling basis is central perspective geometry. The collinearity equations expressing the relationship between object point $i$ appearing in image $j$ for spectral band $k \in\{B, G, R\}$ are given by

$$
\begin{aligned}
& x_{i j k}+\hat{v}_{x_{j k}}=x_{p_{j k}}-c_{j k} \frac{U_{i j j}}{W_{i j}}+\Delta x_{i j k} \\
& y_{i j k}+\hat{v}_{y_{i j k}}=y_{p_{j k}}-c_{j k} \frac{V_{i j}}{W_{i j}}+\Delta y_{i j k}
\end{aligned}
$$

where $(\mathrm{x}, \mathrm{y})$ are the image point measurements, $\left(\mathrm{v}_{\mathrm{x}}\right.$ and $\left.\mathrm{v}_{\mathrm{y}}\right)$ are the image point residuals, $\left(x_{p}, y_{p}\right)$ the principal point coordinates, $\mathrm{c}$ is the principal distance, $(\mathrm{X}, \mathrm{Y}, \mathrm{Z})$ are the coordinates of object point, $(\mathrm{X}, \mathrm{Y}, \mathrm{Z})^{\mathrm{c}}$ are the coordinates of the perspective centre, $\mathbf{M}$ is the rotation matrix from object space to image space that is parameterized in terms of the three Cardan angles $\omega, \phi$ and $\kappa$, and

$$
\left(\begin{array}{c}
U \\
V \\
W
\end{array}\right)_{i j}=\mathbf{M}\left(\left(\begin{array}{c}
X \\
Y \\
Z
\end{array}\right)_{i}-\left(\begin{array}{l}
X \\
Y \\
Z
\end{array}\right)_{j}^{c}\right)
$$

\subsection{Image Distortion Models}

The $\Delta \mathrm{x}_{\mathrm{ijk}}$ and $\Delta \mathrm{y}_{\mathrm{ijk}}$ terms of Equation 1 comprise the sum of image point coordinate corrections for radial lens distortion $\left(\Delta \mathrm{x}_{\mathrm{r}_{\mathrm{k}}}, \Delta \mathrm{y}_{\mathrm{r}_{\mathrm{k}}}\right)$ and decentring lens distortion $\left(\Delta \mathrm{x}_{\mathrm{d}_{\mathrm{k}}}, \Delta \mathrm{y}_{\mathrm{d}_{\mathrm{k}}}\right)$ for spectral band, k. For the Ladybug 5 cameras, the severe barrel distortion requires a five-term radial lens distortion model:

$$
\begin{aligned}
& \Delta x_{r_{k}}=\bar{x}\left(k_{1_{k}} r^{2}+k_{2_{k}} r^{4}+k_{3_{k}} r^{6}+k_{4_{k}} r^{8}+k_{5_{k}} r^{10}\right) \\
& \Delta y_{r_{k}}=\bar{y}\left(k_{1_{k}} r^{2}+k_{2_{k}} r^{4}+k_{3_{k}} r^{6}+k_{4_{k}} r^{8}+k_{5_{k}} r^{10}\right)
\end{aligned}
$$


Although in-air calibration of the GoPro Hero 5 also requires five radial lens distortion terms (Jarron et al, 2019c), only the first three were needed for the in-water calibrations performed herein. The standard model for decentring lens distortion was utilized for both cameras.

$$
\begin{aligned}
& \Delta \mathrm{x}_{\mathrm{d}_{\mathrm{k}}}=\mathrm{p}_{\mathrm{l}_{\mathrm{k}}}\left(\mathrm{r}^{2}+2 \overline{\mathrm{x}}^{2}\right)+2 \mathrm{p}_{2_{\mathrm{k}}} \overline{\mathrm{xy}} \\
& \Delta \mathrm{y}_{\mathrm{d}_{\mathrm{k}}}=\mathrm{p}_{2_{\mathrm{k}}}\left(\mathrm{r}^{2}+2 \overline{\mathrm{y}}^{2}\right)+2 \mathrm{p}_{\mathrm{I}_{\mathrm{k}}} \overline{\mathrm{xy}}
\end{aligned}
$$

(Note that the $\mathrm{i}$ and $\mathrm{j}$ subscripts have been dropped for the sake of clarity.)

\subsection{Constraint Models}

In Case 3, the exterior orientation parameters of the three bands for image $\mathrm{j}$ are constrained to be equivalent. Six independent constraints can be formed between any two bands, so there are 12 constraints for the $\mathrm{B}, \mathrm{G}, \mathrm{R}$ triplet of images for a given image $\mathrm{j}$. The exterior orientation equivalence constraints can be formulated several ways. In terms of the position elements, the approach taken here is to constrain the base vector between two bands to equal zero. For example, the constraint between the blue and green images is given by

$$
\left(\begin{array}{l}
0 \\
0 \\
0
\end{array}\right)+\left(\begin{array}{c}
\hat{\mathrm{v}}_{\mathrm{b}_{\mathrm{X}}} \\
\hat{\mathrm{v}}_{\mathrm{b}_{\mathrm{Y}}} \\
\hat{\mathrm{v}}_{\mathrm{b}_{\mathrm{Z}}}
\end{array}\right)_{\mathrm{BG}}=\mathbf{M}_{\mathrm{B}}\left(\begin{array}{c}
\mathrm{X}_{\mathrm{G}}^{\mathrm{c}}-\mathrm{X}_{\mathrm{B}}^{\mathrm{c}} \\
\mathrm{Y}_{\mathrm{G}}^{\mathrm{c}}-\mathrm{Y}_{\mathrm{B}}^{\mathrm{c}} \\
\mathrm{Z}_{\mathrm{G}}^{\mathrm{c}}-\mathrm{Z}_{\mathrm{B}}^{\mathrm{c}}
\end{array}\right)
$$

The components are expressed in the image space of one of the two bands. The blue band has been arbitrarily chosen here. A similar equation is also written between the blue and red bands or, equivalently, between the green and red bands. For the angular elements of the exterior orientation, the relative angles between the blue and green bands are constrained to equal zero:

$$
\begin{aligned}
& 0+\hat{\mathrm{v}}_{\Delta \omega_{\mathrm{BG}}}=\arctan \left(\frac{-\Delta \mathrm{m}_{32}}{\Delta \mathrm{m}_{33}}\right) \\
& 0+\hat{\mathrm{v}}_{\Delta \phi_{\mathrm{BG}}}=\arcsin \left(\Delta \mathrm{m}_{31}\right) \\
& 0+\hat{\mathrm{v}}_{\Delta \mathrm{B}_{\mathrm{BG}}}=\arctan \left(\frac{-\Delta \mathrm{m}_{21}}{\Delta \mathrm{m}_{11}}\right)
\end{aligned}
$$

where the $\Delta \mathrm{m}_{\mathrm{ij}}$ elements are from the relative rotation matrix between the blue and green bands.

$$
\Delta \mathbf{M}_{\mathrm{BG}}=\mathbf{M}_{\mathrm{G}} \mathbf{M}_{\mathrm{B}}^{\mathrm{T}}
$$

As with the base vector constraints, similar constraints are formed for the blue and red bands.

In Case 4, certain interior orientation elements are constrained to be equivalent. At the time of writing, only the constraints to enforce equivalence of the principal point coordinates have been implemented. The constraint between the blue and green bands is given by

$$
\left(\begin{array}{l}
0 \\
0
\end{array}\right)+\left(\begin{array}{l}
\hat{v}_{\mathrm{x}_{\mathrm{p}}} \\
\hat{\mathrm{v}}_{\mathrm{y}_{\mathrm{p}}}
\end{array}\right)_{\mathrm{BG}}=\left(\begin{array}{l}
\mathrm{x}_{\mathrm{p}} \\
\mathrm{y}_{\mathrm{p}}
\end{array}\right)_{\mathrm{B}}-\left(\begin{array}{l}
\mathrm{x}_{\mathrm{p}} \\
\mathrm{y}_{\mathrm{p}}
\end{array}\right)_{\mathrm{G}}
$$

Similar equations are written between the blue and red bands.

\subsection{Adjustment Models}

The parametric or Gauss-Markov adjustment model was utilized for all four self-calibration models. All interior orientation parameters were modelled as network invariant. The aforementioned constraints were implemented as weighted constraints according to the unified approach to least squares (Mikhail, 1976). This approach offers the advantages of ease of implementation and flexibility in terms of selecting constraint weights. Datum definition was by (absolute) inner constraints on object points.

\section{DESCRIPTION OF THE EXPERIMENTS}

\subsection{Cameras}

As mentioned earlier, the first camera system tested was the Ladybug5 camera system. It comprises six Sony ICX655 cameras (2/3" CCD; $3.45 \mu$ m pixel spacing; 2048 x 2448 pixel image size; $4.4 \mathrm{~mm}$ nominal focal length) mounted in a rigid housing. Five cameras (cameras 0-4) are horizontal looking while the sixth (camera 5) looks upward. Ladybug5 imagery can be acquired in several formats. For these experiments, images were taken in JPEG format as would be the case when the Ladybug5 is deployed on a mobile mapping system.

Four GoPro Hero 5 Black cameras (1/2.3" CMOS; $1.53 \mu \mathrm{m}$ pixel spacing; $4000 \times 3000$ pixel captured image size; $2.68 \mathrm{~mm}$ nominal focal length) were also tested. Each camera was placed in a water-tight acrylic housing with planar port prior to submersion in a fresh water tank for image acquisition. The refraction effects due to the planar port and water increased the nominal focal length by approximately the index of refraction of water to $3.56 \mathrm{~mm}$ (Fryer and Fraser, 1986).

\subsection{Calibration Networks}

The Ladybug5 system was calibrated in an indoor facility at the University of Calgary (Figure 2). This controlled environment $(11 \mathrm{~m} \mathrm{x} 11 \mathrm{~m} \mathrm{x} 4 \mathrm{~m})$ comprises some 500 black-and-white circular targets of varying sizes to permit the calibration of many different types of sensors. For this experiment, 132 targets with $125 \mathrm{~mm}$ radius were used. A total of 232 colour images were captured at three nominal locations within the room. Elements of strong first-order design including convergent imagery, orthogonal roll angles and filling the image format with observations were incorporated into the network. The convergent geometry was easily achieved for the horizontal-looking cameras by rotating the camera system about its vertical axis and acquiring images at each orientation. This also provided the roll diversity for the zenith-looking camera. However, the desired highly-convergent imaging geometry and roll diversity could not be achieved for the upward looking camera and horizontal cameras, respectively. The images were separated into red, green and blue channel images for and the target centre coordinates were measured using the ellipse-fitting algorithm reported in Jarron et al (2019b).

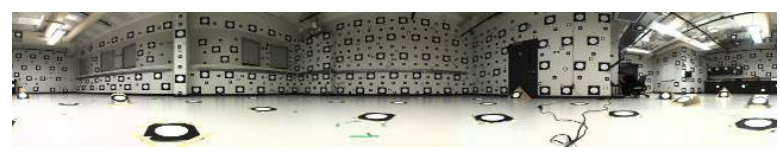

Figure 2. Indoor calibration room at the University of Calgary. 
The GoPro Hero 5 cameras were calibrated underwater in a tank (3 m x $2 \mathrm{~m} \times 1.5 \mathrm{~m}$ deep) at Curtin University (Figure 3). Comprising some 133 white targets $(5 \mathrm{~mm}$ diameter) on a black background, it is an enlarged version of the test field reported in Helmholz and Lichti (2019). The GoPro cameras were set to time-lapse photo acquisition mode then completely submerged in the tank for image capture. The cameras were moved around the calibration frame whilst maintaining a constant standoff distance. Sixteen convergent images with roll diversity were captured. Target centre measurements were made with the centroid method in the Australis software (version 8.33).

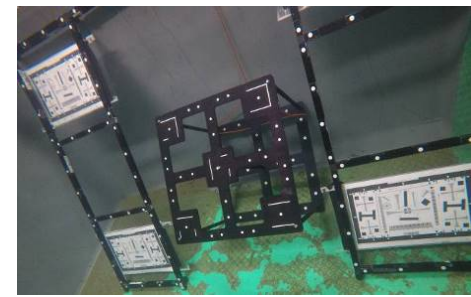

Figure 3. Underwater calibration field at Curtin University.

\subsection{Self-Calibration Adjustments}

The four self-calibrating bundle adjustments cases described in Section 1.2 were performed for each dataset. Since the six cameras of the Ladybug5 comprise an integrated system, they were calibrated in the same adjustment for each case. However, constraints enforcing the stability of the relative orientation parameters between camera pairs of the Ladybug5 system were not included for this experiment. The GoPro cameras were calibrated in separate adjustments.

\section{EXPERIMENT RESULTS}

\subsection{Adjustment Summaries}

Prior to analysing the interior orientation parameters, it is worth presenting some of the bundle adjustment metadata to provide an understanding of the overall redundancy, the fit of the observations to the model and the achieved precision. For the sake of brevity, only the metadata for the Case 2 adjustment are shown (Table 1). The overall degrees-of-freedom (df) for the Ladybug5 is much higher because all six cameras were calibrated together. Mean values from the four cameras are reported for the GoPro. In each case, the fit of the observations to the model $(\mathrm{rmsxy})$ is approximately $1.5 \mu \mathrm{m}$ and mean object space coordinate precision $\left(\bar{\sigma}_{\mathrm{c}}\right)$ is sub-millimetre.

\subsection{Principal Distance Behaviour}

Principal distance (PD) estimates and $95 \%$ confidence regions for the Ladybug 5 cameras are presented in Figure 4. The independent adjustments (Case 1; Figure 4a) show the existence of longitudinal chromatic aberration as the PDs differ between the bands. No consistent pattern exists between the PDs of the different spectral bands among the six cameras and the range of estimates is up to $3.5 \mu \mathrm{m}$ ( 1 pixel). The precision of the horizontal cameras (0-4) is on the order of $0.7 \mu \mathrm{m}(\sim 0.2$ pixels). The precision of the vertical camera (5) is lower $(1.7 \mu \mathrm{m}, 0.5$ pixels) due to the lack of convergent geometry. Since only the B and R bands' PDs for camera 4 are statistically different, the differences are likely due to random errors.

\begin{tabular}{|l|c|c|}
\hline & Ladybug5 & GoPro Hero 5 (mean) \\
\hline $\mathrm{df}$ & 32545 & 5721 \\
\hline $\mathrm{rmS}_{\mathrm{xy}}(\mu \mathrm{m})$ & 1.7 & 1.5 \\
\hline $\bar{\sigma}_{\mathrm{c}}(\mathrm{mm})$ & 0.7 & 0.4 \\
\hline
\end{tabular}

Table 1. Case 2 adjustment data

The effects of the combined adjustment (Case 2, Figure 4b) are minimal. The estimates and precision for the horizontal cameras do not change significantly. The vertical camera PDs are affected more, up to $1.6 \mu \mathrm{m}(0.45$ pixels $)$ in terms of the estimate and the precision improves to $1.4 \mu \mathrm{m}$ ( 0.4 pixels). Addition of the exterior orientation equivalence constraints (Case 3, Figure 4c) influences some of the PDs, in particular for cameras 2 and 5, but the differences between spectral bands are still insignificant except for the aforementioned example. Not surprisingly, imposing the principal point equivalence constraints (Case 4, Figure 4d) has no effect on the PD.

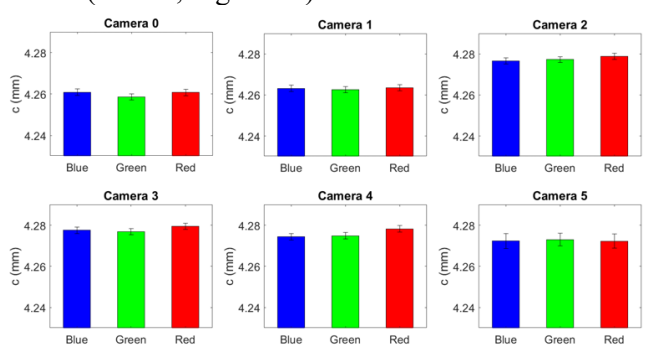

a)

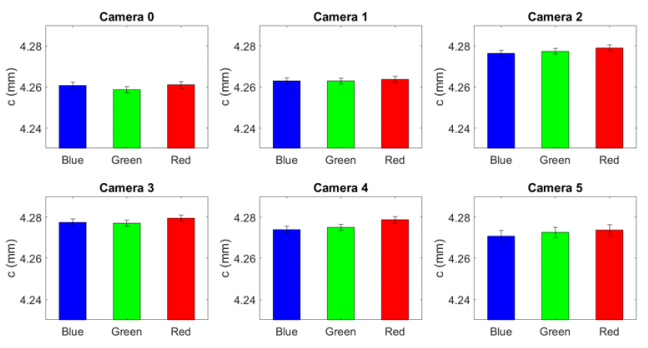

b)

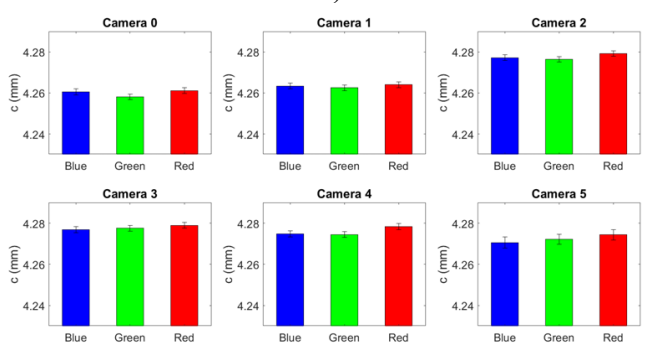

c)

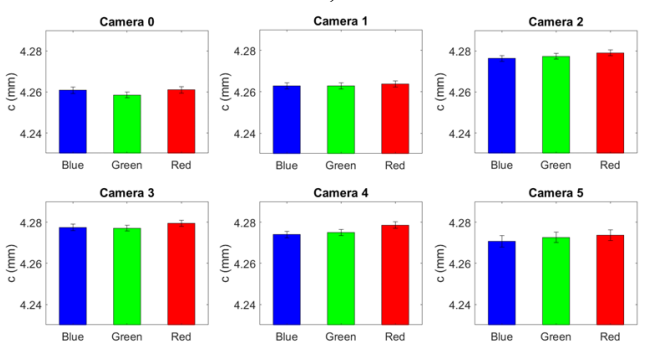

d)

Figure 4. Ladybug 5 principal distances and $95 \%$ confidence intervals. a) Case 1, b) Case 2, c) Case 3, d) Case 4. 

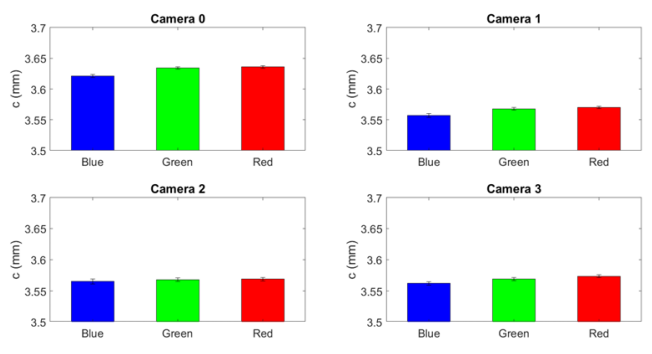

a)
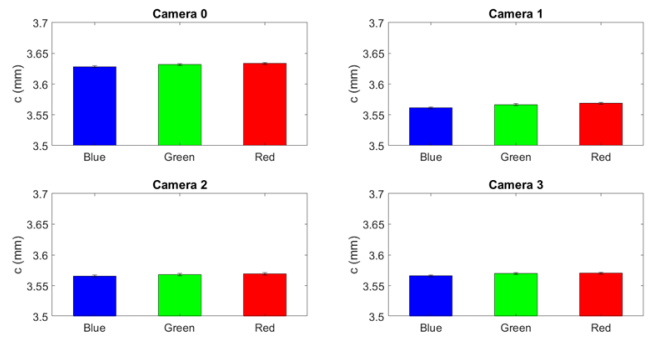

b)
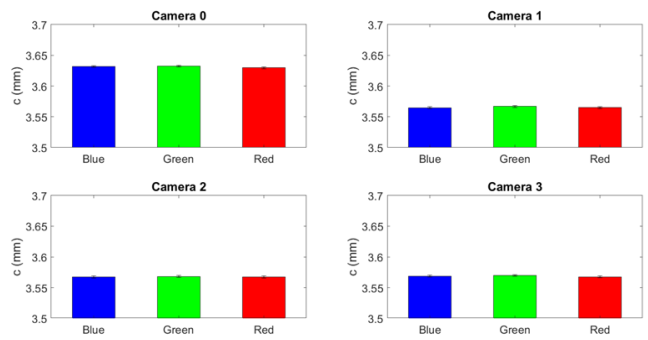

c)
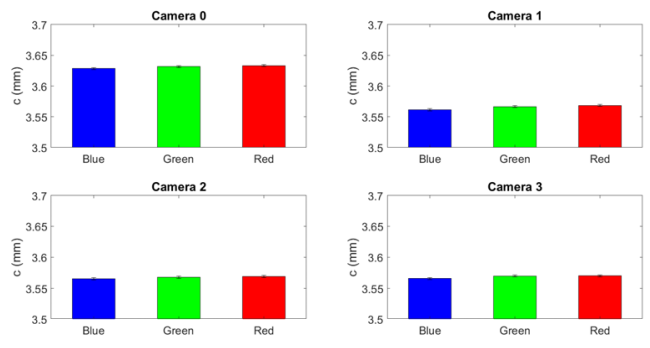

d)

Figure 5. GoPro Hero 5 principal distances and $95 \%$ confidence intervals. a) Case 1, b) Case 2, c) Case 3, d) Case 4.

Figure 5 provides the PDs and $95 \%$ confidence regions for the GoPro Hero 5 cameras. For Case 1 (Figure 5a), the range of estimates between bands for a given camera is up to $14.8 \mu \mathrm{m}$ (9.7 pixels; camera 0). In general, the blue estimate is shortest while the red is the longest, which follows the aforementioned theoretical expectation. Precision estimates range from $1.1 \mu \mathrm{m}$ to $2.0 \mu \mathrm{m}$ ( 0.7 to 1.3 pixels). Statistically significant differences between pairs of bands exist in three of eight cases. The combined adjustments (Case 2, Figure 5b) differ in that the differences among bands are less pronounced. Although the hierarchy among bands still exists, their range is more uniform, with camera 1 having the largest range of $7.3 \mu \mathrm{m}$ (4.8 pixels). Despite the smaller range of principal distance estimates, four of the eight pairs are statistically different. The improved precision ( 0.8 to $0.9 \mu \mathrm{m} ; 0.5$ to 0.6 pixels $)$ is certainly a contributing factor. Enforcement of the exterior orientation constraints (Case 3, Figure 5c) reduces the differences between bands even more, with the maximum being $2.6 \mu \mathrm{m}$ (1.7 pixels). Furthermore, the hierarchical relationship seen in the earlier two cases no longer exists. The green band features the longest principal distance for all four cameras. There is no change to the precision. Only one pair of PDs is significantly different, so the differences in this adjustment case are also likely due to random errors. As with the Ladybug5 cameras, the Case 4 (Figure 5d) and Case 1 results are effectively the same.

\subsection{Principal Point Behaviour}

The principal point (PP) estimates for each spectral band for all six Ladybug5 cameras along with $95 \%$ confidence ellipses are shown in Figure 6. The coordinates are referenced to the corner of the image format. As can be seen for Case 1 (Figure 6a), differences of up to $2.4 \mu \mathrm{m}$ ( 0.7 pixels) exist, but they are not statistically significant. The vertical camera (5) shows the tightest clustering of PP estimates. The precision estimates for the horizontal cameras is heterogeneous, about $1 \mu \mathrm{m}(0.3$ pixels $)$ in $\mathrm{x}$ and $0.6 \mu \mathrm{m}(0.18$ pixels $)$ in $\mathrm{y}$, due to the lack of roll diversity mentioned earlier. It is uniform at about $0.4 \mu \mathrm{m}(0.1$ pixels) for the vertical camera due to the roll diversity. The combined adjustment (Case 2, Figure 6b) slightly decreases the spread of PP estimates to $2.1 \mu \mathrm{m}$ ( 0.6 pixels). The horizontal camera precision benefits the most, improving to $0.7 \mu \mathrm{m}$ and $0.6 \mu \mathrm{m}(0.2$ pixels and 0.16 pixels $)$ in $\mathrm{x}$ and $\mathrm{y}$, respectively. The exterior orientation constraints (Case 3, Figure 6c) further reduce the range of $\mathrm{PP}$ estimates to $0.3 \mu \mathrm{m}(0.1$ pixels $)$ or less. Precision is further improved to $0.6 \mu \mathrm{m}$ and $0.3 \mu \mathrm{m}(0.16$ and 0.1 pixels) in $\mathrm{x}$ and $\mathrm{y}$, respectively, for the horizontal cameras and to $0.2 \mu \mathrm{m}$ ( 0.06 pixels) for the vertical camera. The PP equivalence constraints (Case 4; Figure 6d) eliminate all between-band PP coordinate differences and realize the same precision that the exterior orientation constraints (Case 3) achieved.
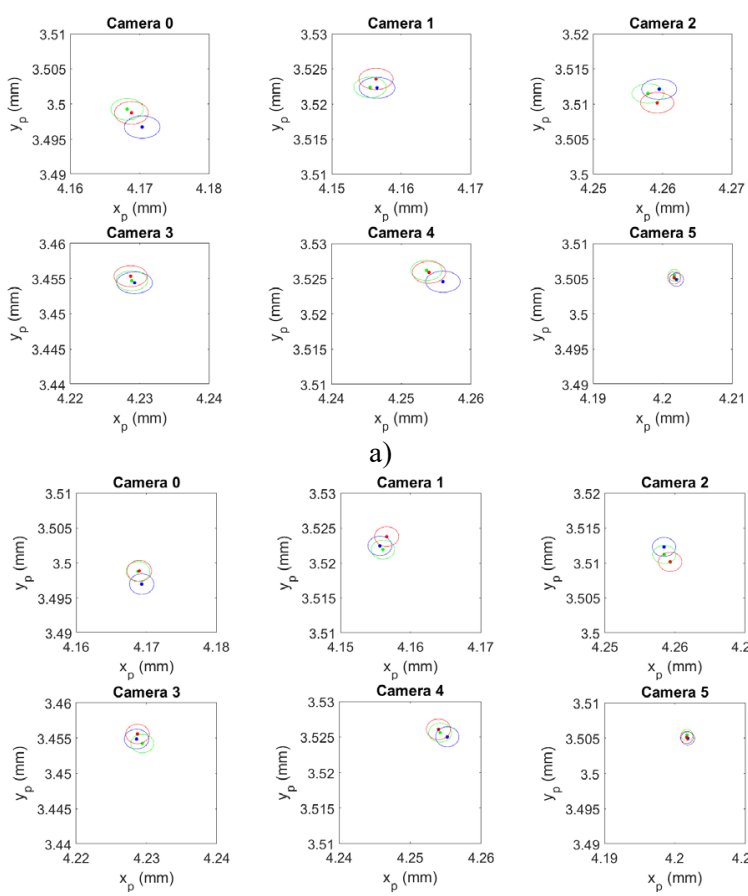

a)
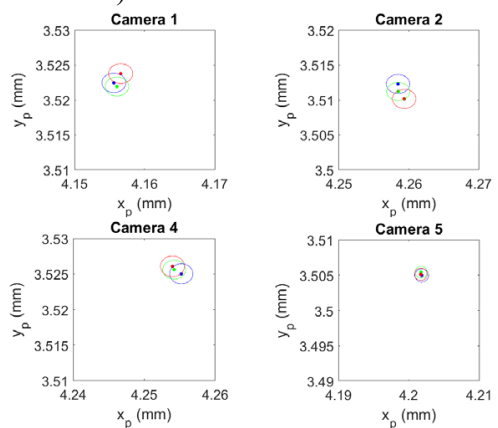

b) 

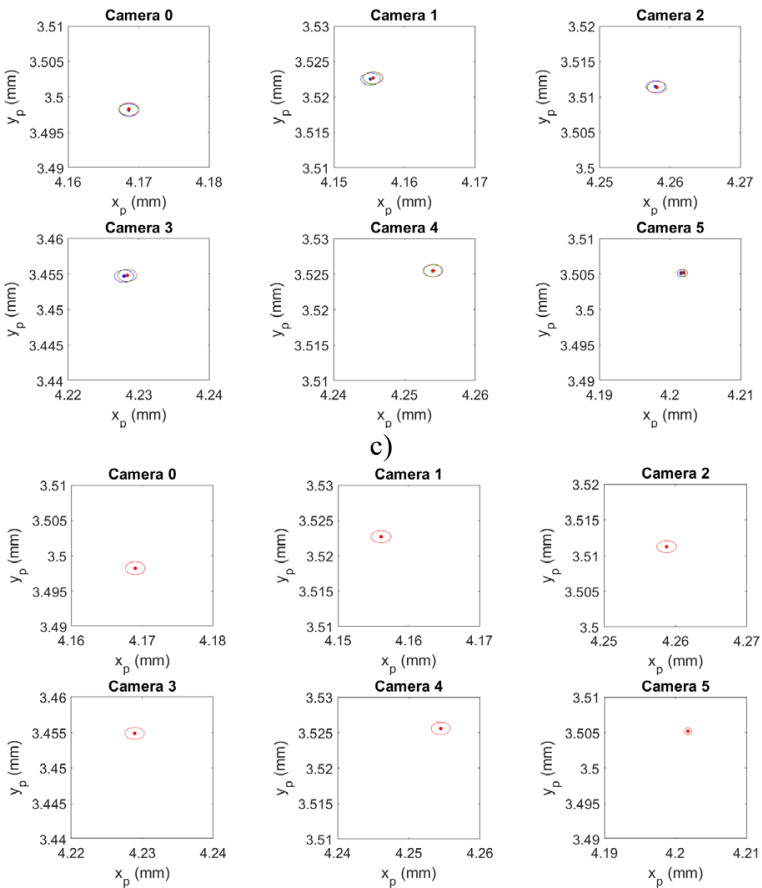

d)

Figure 6. Ladybug5 principal point and $95 \%$ confidence region for a) Case 1, b) Case 2, c) Case 3, d) Case 4

The PP estimates and confidence regions are shown for the GoPro Hero 5 cameras in Figure 7. In this case, the coordinates are referenced to the image centre. The Case 1 (Figure 7a) differences among different colour band PP estimates are generally much larger, up to $17.7 \mu \mathrm{m}$ (11.6 pixels) and several are statistically different. Overall, the precision is more homogeneous but varies considerably from one camera to the next $0.9 \mu \mathrm{m}$ to $2.9 \mu \mathrm{m}$ ( 0.6 pixels to 1.9 pixels). The combined adjustment (Case 2, Figure 7b) reduces the spread of PP estimates to a maximum of $6.0 \mu \mathrm{m}$ ( 3.9 pixels). The precision is also improved, quite considerably in some cases, ranging from $0.9 \mu \mathrm{m}$ to $1.4 \mu \mathrm{m}$ ( 0.6 to 0.9 pixels). The exterior orientation constraints (Case 3, Figure 7c) further reduce the spread to a maximum of $0.2 \mu \mathrm{m}(0.15$ pixels $)$ and further improve precision to $0.6 \mu \mathrm{m}$ to $1.1 \mu \mathrm{m}$ ( 0.4 to 0.7 pixels). Finally, as with the Ladybug5, the PP constraints (Case 4, Figure 7d) eliminate the PP coordinate differences and achieve a precision comparable to that obtained with the exterior orientation constraints.
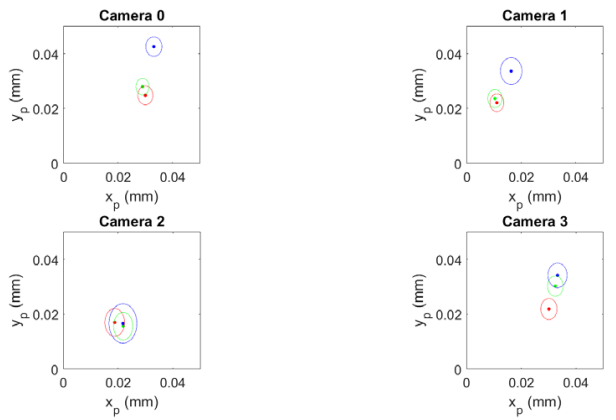

a)
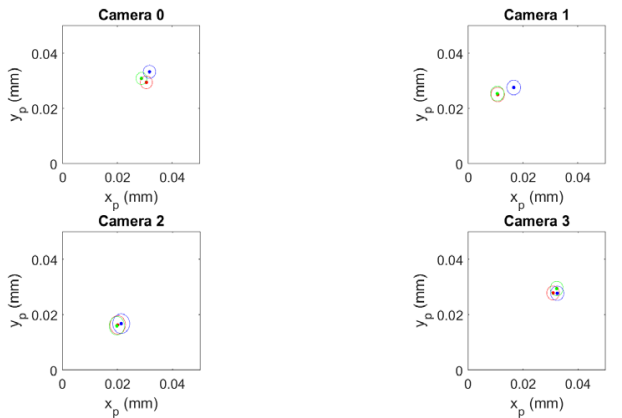

b)
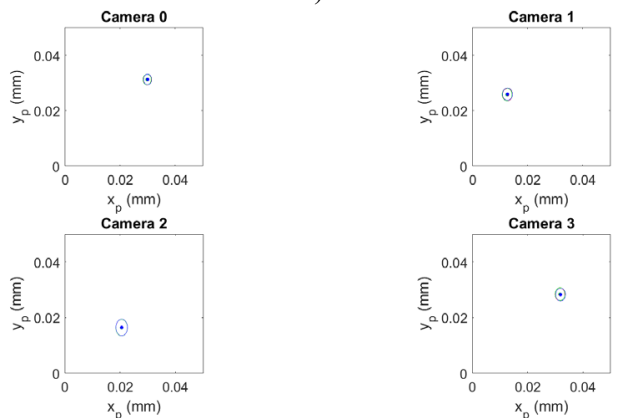

c)
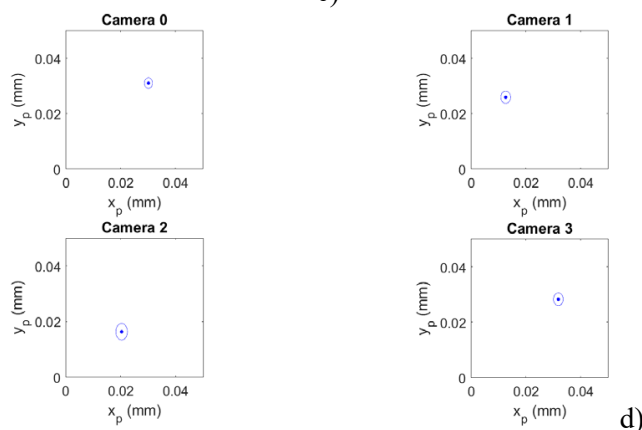

Figure 7. Go Pro Hero 5 principal point and $95 \%$ confidence region for a) Case 1, b) Case 2, c) Case 3, d) Case 4.

\subsection{Radial Lens Distortion Behaviour}

As mentioned earlier, the Ladybug5 cameras exhibit a high amount of radial lens distortion: nearly 2000 pixels at the corner of the image format. An example is provided in Figure 8. As can be seen, it is difficult to discern differences between the profiles of the three colour bands. Therefore, instead of plotting the actual distortion profiles, differences between profiles are plotted (Figure 9). The differences are computed relative to the mean of the three profiles for a given camera for each adjustment case. Thus, only relative agreement among the profiles of different bands can be inferred.

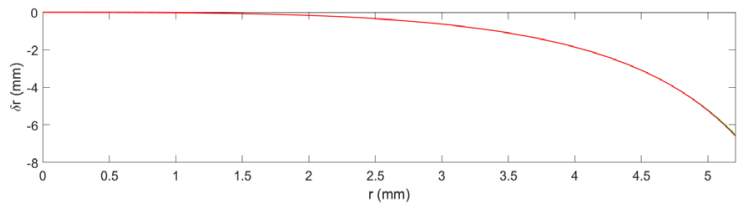

Figure 8. Ladybug5 radial lens distortion profiles for camera 0 , Case 1. 


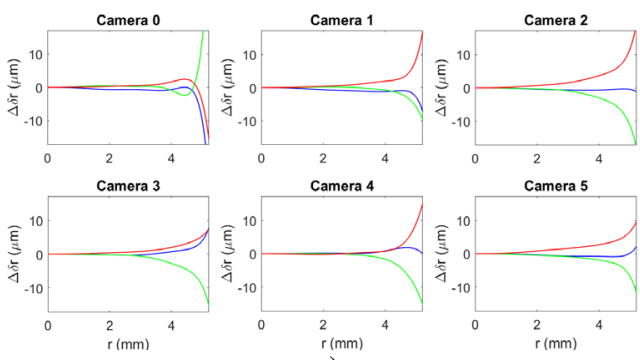

a)
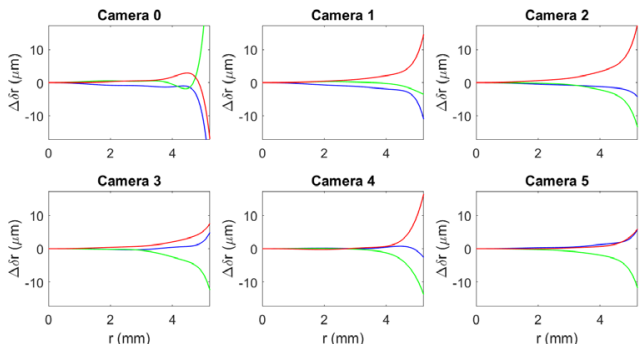

b)
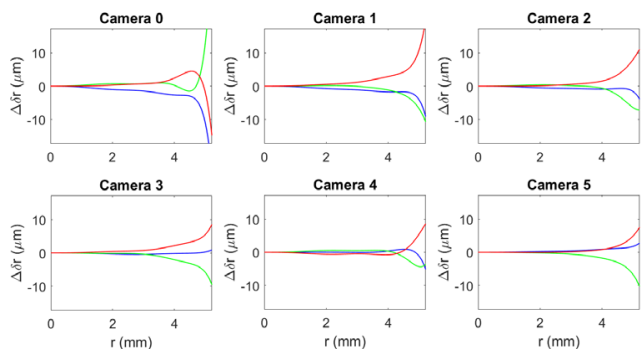

c)
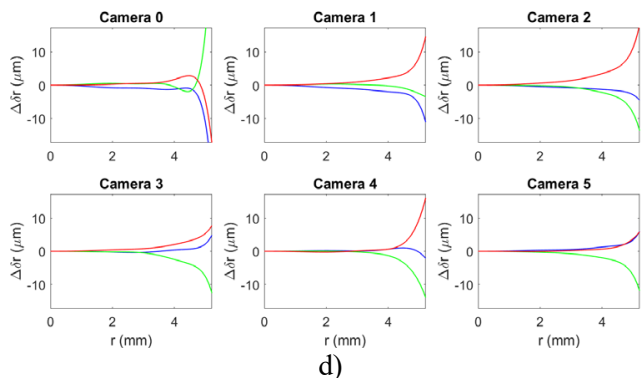

Figure 9. Ladybug5 radial lens distortion profile differences from the mean of all three bands for a) Case 1, b) Case 2, c) Case 3, d) Case 4.

The profile differences of the independent adjustments (Case 1, Figure 9a) show very close agreement (a few $\mu \mathrm{m}$ or less) up to a radial distance of $4 \mathrm{~mm}$, which is $75 \%$ of the image format. Beyond this point, the curves depart more considerably. This pattern repeats for all constrained cases (Figures 9b-9d) with only minor variations, suggesting that the radial distortion curves are independent of the type of adjustment performed.

The in-water GoPro Hero 5 radial lens distortion is much less severe than that of the Ladybug5 cameras, so the actual distortion profiles are plotted (Figure 10). In Case 1 (Figure 10), all four cameras exhibit the same behaviour in terms of the hierarchy of curves and curve behaviour. The red-channel barrel distortion reaches a maximum of approximately $80 \mu \mathrm{m}$ ( $>50$ pixels). The profile changes sign near the edge of the format where the blue channel reaches a maximum of up to 45 $\mu \mathrm{m}$ ( $\sim 30$ pixels). The curves exhibit clear differences of $15 \mu \mathrm{m}$
( $\sim 10$ pixels) due to transverse chromatic aberration. There are no discernible differences between the profiles of the other four cases, so the corresponding figures have been omitted. Thus, as with the Ladybug5 cameras, radial lens distortion seems to be independent of the adjustment case.
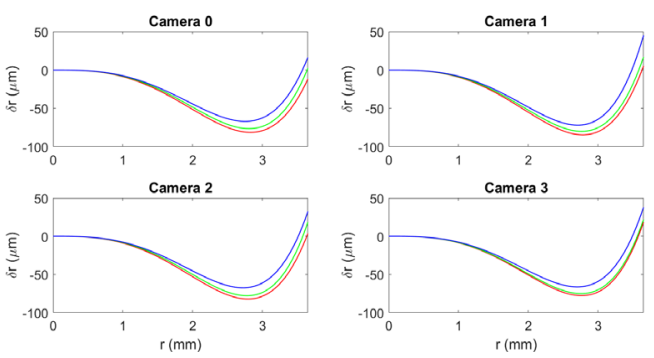

Figure 10. Case 1 GoPro Hero 5 radial lens distortion profiles.

\subsection{Decentring Distortion Behaviour}

Decentring profiles for Case 1 of the Ladybug5 cameras are given in Figure 11. Overall, the amount of distortion is low, reaching a maximum of about $6 \mu \mathrm{m}(<2$ pixels $)$ for one camera. Four of the remaining five reach $4 \mu \mathrm{m}(\sim 1$ pixel $)$ or less. There is no clear hierarchy of the bands and the differences between them are less than $1 \mu \mathrm{m}$ at the format extents. The differences among the different adjustment cases (not shown) are less than $1 \mu \mathrm{m}$ and the behaviour is not consistent. In some cases, the B, $\mathrm{G}$ and $\mathrm{R}$ curves become more tightly clustered while in others they do not.
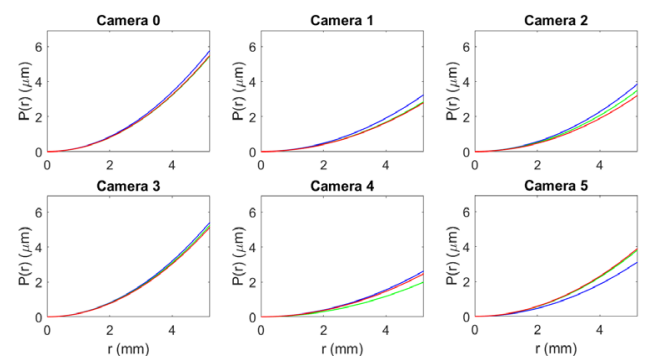

Figure 11. Case 1 Ladybug5 decentring lens distortion profiles.

Case 1 decentring profiles for the GoPro cameras are given in Figure 12. The amount of distortion is also low, reaching a maximum of almost $10 \mu \mathrm{m}(\sim 6.5$ pixels $)$ for one camera, while the others only reach $5 \mu \mathrm{m}$ ( $\sim 3$ pixels) or less. Once again, there is no clear hierarchy of the bands and the differences between them are less than $1 \mu \mathrm{m}$ at the format extents except for camera 1. The addition of the various constraints of the other adjustment cases (not shown) does cause the curves to generally cluster together more.
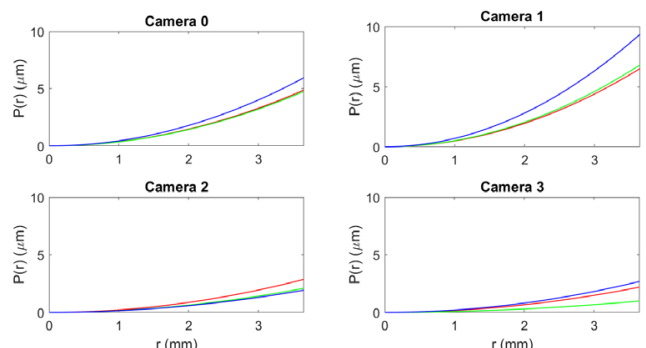

Figure 12. Case 1 Go Pro Hero 5 decentring lens distortion profiles. 


\section{CONCLUSIONS}

A set of self-calibration experiments on two types of colour digital cameras has been performed to quantify the amount of chromatic aberration present and to examine the impacts of various types of geometric constraints. The six Ladybug5 cameras exhibited a low amount of transverse chromatic aberration (on the order of $1-2$ pixels). The longitudinal chromatic aberration was not statistically significant. The observed differences in principal distance were attributed to random errors. The GoPro Hero 5 cameras exhibited a large amount of transverse chromatic aberration (on the order of 25 pixels). Longitudinal chromatic aberration was also observed as there were some significant differences in principal distance. A clear hierarchy among spectral bands that generally followed theoretical expectations was found for the unconstrained and combined adjustment, cases. This was not observed for the case with constrained exterior orientation parameters.

The combined self-calibration enforces the constraint of common object point coordinates on the adjustment of the observations from the three spectral bands. For the Ladybug5, this had only minimal effect on the principal distances while for the GoPro cameras the differences between spectral band principal distances was reduced. For both cameras, these constraints reduced the spread of principal point locations between bands and improved principal point precision. The exterior orientation equivalence constraints had little effect on the Ladybug5 principal distances. The differences between spectral principal distances were reduced for the GoPro cameras, but the aforementioned hierarchy was preserved. For both cameras, the spread of principal point estimates was further reduced and the precision was improved more. The principal point equivalence constraints accomplished their intention and improved precision to the same level achieved by the exterior orientation constraints. The radial lens distortion was observed to be unaffected by adjustment case. The decentring distortion showed no patterns as a function of adjustment case for the Ladybug5 whereas for the GoPro cameras the profiles were slightly more tightly clustered together in some cases.

Several avenues for further research exist. Although the behaviour of radial and decentring distortion has been investigated, it has not been contextualized with the estimated precision of the profiles. Furthermore, the behaviour of the exterior orientation parameters as a function of the different adjustment constraints has not been reported. Finally, the impact of adjustment case on $3 \mathrm{D}$ reconstruction accuracy needs to be investigated.

\section{ACKNOWLEDGEMENTS}

Funding for this research was provided by the Natural Sciences and Engineering Research Council of Canada, the University of Calgary, McElhanney Geomatics Engineering Ltd and the Curtin Institute for Computation. The Curtin University Centre for Marine Science and Technology is thanked for providing access to the tank.

\section{REFERENCES}

Cronk, S, Fraser C, Hanley, H (2006) Automated metric calibration of colour digital cameras. Photogrammetric Record. 21 (116), 355-372.
Fryer, JG, Fraser, CS (1986) On the calibration of underwater cameras. Photogrammetric Record. 12 (67) 73-85.

Helmholz, P, Lichti, DD (2019). Assessment of chromatic aberrations for GoPro 3 cameras in underwater environments. ISPRS Annals of Photogrammetry, Remote Sensing and Spatial Information Sciences, IV-2/W5, 575-582.

Jarron, D, Lichti, DD, Shahbazi, M, Radovanovic, R (2019a). Multi-Camera Panoramic Imaging System Calibration. In Proceedings of the $11^{\text {th }}$ International Conference on Mobile Mapping. Shenzhen, China, 6-8 May. 70-77.

Jarron, D, Shahbazi, M, Lichti, D, Radovanovic, R (2019b) Automatic detection and labelling of photogrammetric control points in a calibration test field. International Archives of Photogrammetry, Remote Sensing and Spatial Information Sciences, XLII-2/W13, 1673-1680.

Jarron, D, Shahbazi, M, Lichti, D, Radovanovic, R (2019c) Modelling wide-angle lens cameras for metrology and mapping applications. ISPRS Annals of Photogrammetry, Remote Sensing and Spatial Information Sciences, IV-2/W7, 79-86

Luhmann, T, Hastedt, H, Tecklenburg, W (2006). Modelling of chromatic aberration for high precision photogrammetry. Image Engineering and Vision Metrology, International Archives of the Photogrammetry, Remote Sensing and Spatial Information Sciences, 36, 173-178.

Matsuoka, R, Asonuma, K, Takahashi, G, Danjo, T, Hirana, K (2012). Evaluation of correction methods of chromatic aberration in digital camera images, ISPRS Annals of Photogrammetry, Remote Sensing and Spatial Information Sciences, I-3, 49-55.

Menna, F, Nocerino, E, Remondino, F (2017). Flat versus hemispherical dome ports in underwater photogrammetry. The International Archives of the Photogrammetry, Remote Sensing and Spatial Information Sciences, Volume XLII-2/W3, 481487.

Mikhail, EM (1976) Observations and Least Squares. IEP, 497 pp.

Pöntinen, P (2008) Study on chromatic aberration of two fisheye lenses. The International Archives of the Photogrammetry, Remote Sensing and Spatial Information Sciences, Volume XXXVI, Part B3a, 27-32

Ray, SF (1994). Applied Photographic Optics. Focal Press, Oxford. 586 pp.

Robson, S., MacDonald, L., Kyle, S.A., Shortis, M.R, (2014). Multispectral calibration to enhance the metrology performance of C-mount camera systems. The International Archives of the Photogrammetry, Remote Sensing and Spatial Information Sciences XL-5, 517-521.

Van Den Heuvel, F.A., Verwaal, R., Beers, B. (2006). Calibration of fisheye camera systems and the reduction of chromatic aberration, The International Archives of the Photogrammetry, Remote Sensing and Spatial Information Sciences, Volume XXXVI, Part 5. 